\title{
Over, Under, In-Between: Worker Housing Within an Industrial Ecology
}

\author{
JASON F. CARLOW \\ American University of Sharjah
}

The research and design work presented in this paper was organized and conducted through an advanced design studio at the Department of Architecture at the American University of Sharjah in the UAE. The premise of the studio was to create new housing units specifically designed for low income workers who are not eligible for corporate worker housing in the UAE and not permitted to live in family designated residential districts. These low income workers often struggle to find affordable housing within the industrial zones of the rapidly growing urban metropolis surrounding Dubai. A key design research question asked how housing could be built on spatially confined sites within an industrial zone and provide not only secure and healthful shelter for the residents, but programs and amenities that build a sense of community as well.

\section{INTRODUCTION}

Affordable housing is a particularly sensitive issue in a country with a large expatriate, working-class population and a large income gap between groups of residents across the socioeconomic spectrum. Although developable land is seemingly limitless in the United Arab Emirates, due to tight governmental control over land policies and a relatively high cost of living, the demand for comfortable, affordable housing for working-class expatriates is great. Many working class individuals are forced to live in cramped, corporately owned dormitories or within industrial areas of the city that offer few public amenities or open space. However, Dubai and other cities in the Gulf region are working on new initiatives to provide better housing for low-income residents. The government of Dubai has recently enacted policies to formally address the socio-economic stratification of income groups and address the desperate need for affordable housing. ${ }^{1}$ The research and design work presented in this paper has been in a sense triggered by recent efforts to address the problems and challenges of housing in rapidly developing cities such as Dubai and neighboring Sharjah. The work represents an effort to identify key issues in the development of affordable housing for the UAE and presents new housing solutions through design iterations.

The title of the fifth year, undergraduate design studio, Over, Under, In-between, therefore not only refers to the activation and occupation of space for housing within the city, but how affordable housing must work above, below and in spite of socio-economic obstacles within the global culture and local space of industrial production. How can architecture move beyond the mere provision of shelter and allow residents opportunities to advance their livelihoods and improve the overall built environment? The pedagogical goals of the studio relate to the establishment of what urban philosopher Richard Sennett refers to as the Open City. "The cities everyone wants to live in should be clean and safe, possess efficient public services, be supported by a dynamic economy, provide cultural stimulation, and also do their best to heal society's divisions of race, class, and ethnicity." 2 Design solutions seek opportunities to offer an open system of public amenities to the industrial zones and social and economic mobility to the residents.

\section{STUDIO CONTEXT}

The gleaming towers, expansive shopping malls, multi-lane highways and luxury villas of the Gulf region are examples of what Sennett would call a "closed" urban system. The closed nature of parts of Dubai and similar cities are in part due to the "over-determination both of the city's visual forms and its social functions." ${ }^{3}$ These closed systems have, ironically, been built and maintained by crews of migrant workers from across the Global South who do not have equal access to the closed city they live in. To further complicate the urban context of the UAE, workers in the construction and service industries in the UAE and other countries have immigrated over the past decades to find work and careers far from home. The number of workers that have immigrated to the UAE far outnumbers the population of permanent citizenry of the Emirates. Cities like Dubai are also built on real estate development, tourism and a finance industries that are fueled by global capital. A very large income gap between the wealthy and the working class has subsequently developed.

Given the nature of the work available and policies that prohibit sponsorship visas for low-income workers' families from living in the Emirates, a large percentage of this working class is relatively young and male. Male expatriate residents who want to bring their families into the country must earn a minimum monthly salary of 4,000 AED (\$1089 USD) ${ }^{4}$ preventing many workers from living together with their families. Subsequently, a significant demographic shift towards large percentages of young men working in the Emirates has skewed the make-up of residential communities and lifestyles. Tendencies of groups of young men to sublet and subdivide residential housing stock to make it more affordable has led to animosity from local residents, families and generally put a strain on housing conditions. 



Figure 1: Reframing Industrial Housing by Farah Kazali and Joanna Fattal (above right) reconfigures the ubiquitous Butler Building frame to hybridize spaces for working and dwelling while the Elevating Industrial Living project (above left) by Mona Moussalem and Maha Abdelsalam suspends leisure and public open spaces above a factory yard.

Housing regulations that control where migrant workers may reside have become stricter in recent years. The Emirate of Sharjah, bordering Dubai, is home to many low-income workers who have sought more affordable housing than Dubai has to offer. In Sharjah, government agencies have forbade young, single, working-class men, commonly referred to as "bachelors," from living in family designated areas. Instead, bachelors are forced to live in industrial areas, surrounded and bound by districts and buildings dedicated to storage, logistics and manufacturing. ${ }^{5}$

In reaction to the lack of public amenities, open spaces and space for leisure, working class bachelors living in industrial zones have constructed, developed and inhabited an informal layer of leisure spaces between factories, in vacant lots, between parking spaces and at the periphery of the city. The informal occupation of the interstitial and peripheral zones of the rapidly developing, twenty first century city by the working class has become a source of inspiration for new sites for and forms of affordable housing.

The test site chosen for the studio was Industrial Areas 10 and 11 in Sharjah, on the border of Dubai. These complex zones of industrial, residential and retail activity are situated relatively close to the American University of Sharjah so that students could have easy access for analysis and mapping. Urban analysis played a key role in the development of housing prototypes for an underprivileged element of society in a complex site. Students were encouraged to investigate dynamic issues inherent in these complex industrial zones based on Andrea Kahn's theories for the definition of urban sites:
Urban sites are dynamic rather than static, porous rather than contained...Defining them in design terms thus does not come down to establishing some unique identity of a limited physical place, but quite the opposite. It involves recognizing the overlay and interplay of multiple realities operating at the same time, on the same place. How designers give definition to these multivalent and multiscalar urban design sites, however, remains an open question. ${ }^{6}$

Analytical maps and diagrams were invented and developed to record patterns of mass, void, flows, movement, exchange, production and inhabitation. Resultant maps and drawings identify social, economic and spatial issues that aided the selection of strategic sites for architectural and urban intervention.

\section{DESIGN PROJECTS}

Projects test new sites, programs and opportunities for affordable housing that may create or activate new types of space within industrial areas. The design of new prototypes for living challenge the typical housing types in the greater Dubai region to respond more thoughtfully to environmental, social, economic, urban and architectural conditions. In an attempt to consolidate the investigations undertaken by the students, projects with similar strategies are grouped and presented in the following categorized sections.

\section{Category 1: Typological Interventions}

The first series of projects investigate and alter traditional building types within Sharjah's industrial zone to create new space for housing. Sharjah's Industrial Areas 10 and 11, contain 


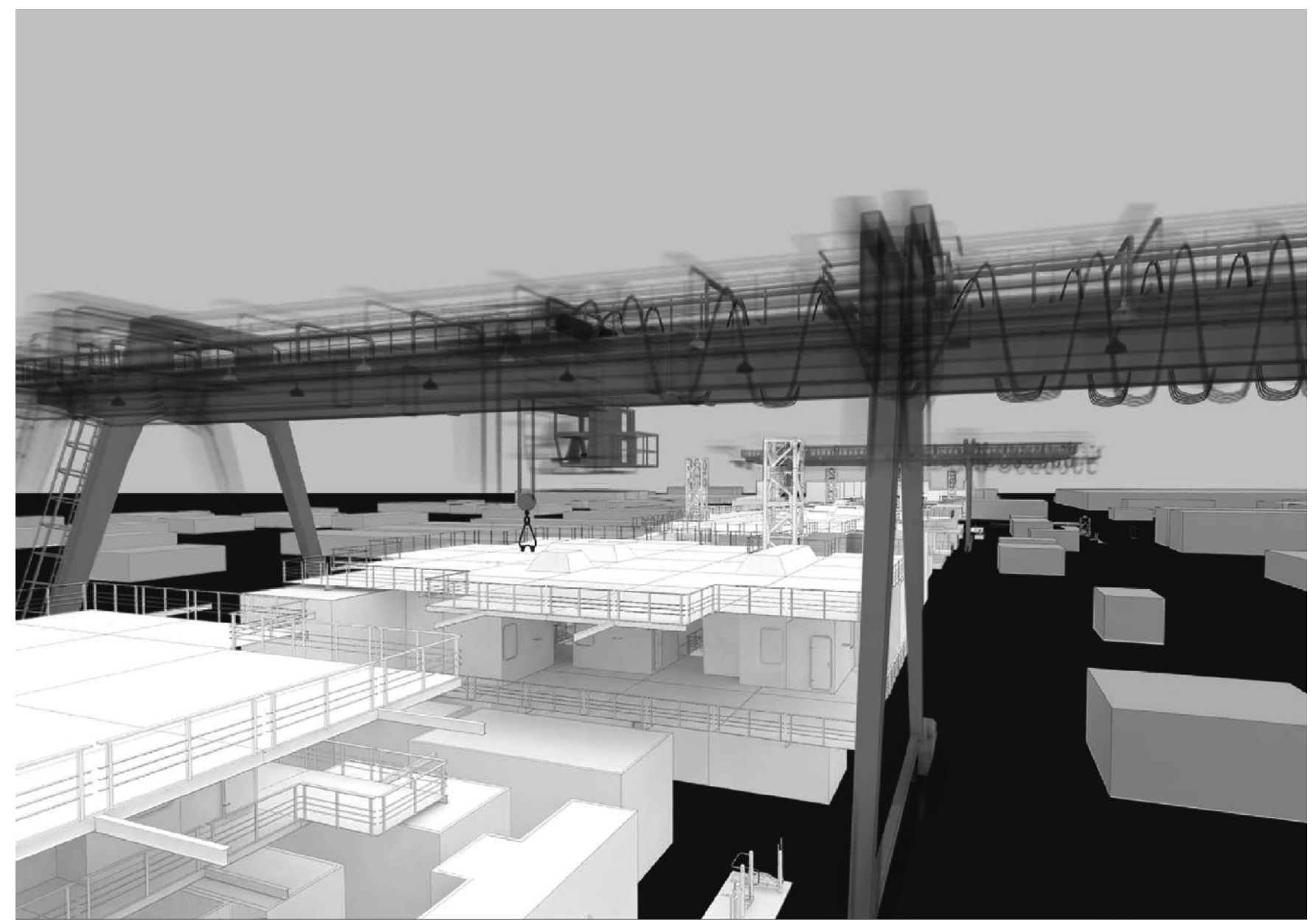

Figure 2: A community formed and adapted by a production line gantry by Bishoy Rami Abd El Nour and Nermin Sherif Hegazy

numerous warehouses that are not only abandoned in the present, but also face the risk of being inadequate spaces in the future. The project entitled, Reframing Industrial Housing by Farah Kazali and Joanna Fattal (figure 1) proposes the repurposing and reconfiguration of the standard Butler Building structural frame to accommodate for current and future programs, economies and urban fabrics. Indoor and outdoor production zones are intertwined with residential units suitable for both residents of the neighborhood and workers in the production zones. The residential zones exist over, under, next to, and in between the frames that formulate the warehouse making the Butler Building frame a site for investigation.

The impact of the project exists on a global scale in that it could be transferred to any industrial context. It can also be assembled in existing or incomplete Butler Building frames. A new building type is developed out of the existing condition by strategically manipulating the frames. Structural adjustments allow for different spatial configurations which subsequently generate the organization of the interior. The aim for this project is to rethink the existing living conditions within the industrial areas by hybridizing production and residential zones in anticipation of future changes in the work/live relationship of the site and the region.

A second project in this category by Mona Moussalem and Maha Abdelsalam is entitled Elevating Industrial Living (figure 1). The team's research and site analysis documented the ad hoc leisure spaces built by the migrant workers of Sharjah. The analysis revealed a need for improved communal spaces for workers within Sharjah's industrial zones. The project therefore seeks to activate vertical air space as a new possibility for housing and public space. The space above and adjacent to an existing residential building was chosen as a project site. The design provides an elevated structure that contains improved residential housing with communal spaces, along with a new factory and production space below. The project utilizes a rigid, cantilevered space frame, into which a number of reconfigurable residential units are placed. Unit re-configurability makes it possible for different programs to plug in and out and be modified according to future programmatic needs over time. A suspended landscape of leisure spaces drops down into the production space below, providing a complex interaction between domesticity 


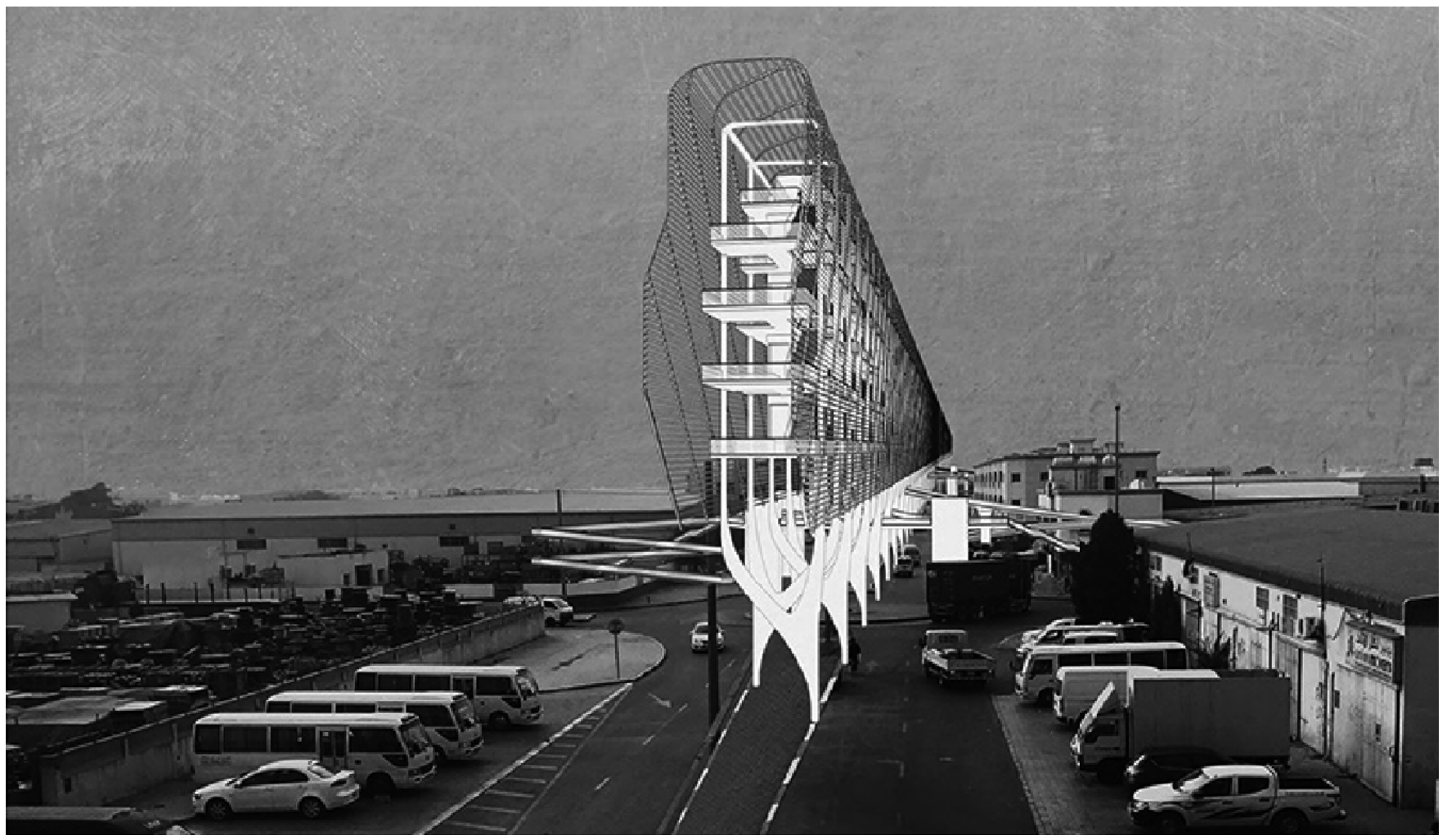

Figure 3: Saskpower Building, interior view of entrance lobby showing original mosaic finishes and illuminated ceiling. Undated, Saskpower Archives

and work zones through the penetration of programmatic volumes. This prototype is designed to be replicated across industrial zones to provide more amenity hubs for foreign and temporary workers.

\section{Category 2: Dwelling with Industrial Artifacts}

The Mechanized Urbanism project by Bishoy Rami Abd EI Nour and Nermin Sherif Hegazy (figure 2) tests the ability of a community to be developed and shaped by the large scale equipment scattered around the typical industial zones. Sharjah's industrial areas are characterized by clusters of production and housing facilities in combination with a scattered array of services and various social nodes. The proposal seeks to develop a reconfigurable building prototype that combines the three aspects of production, housing and leisure, in a setting where they can co-exist and function in a vibrant network. The different components of the project can be reconfigured to create adaptive combinations of housing, production and amenities. The project challenges the separation of traditional housing types and factories in the industrial areas and instead combines living with working together in a dynamic, prototypical community. Incorporating machinery like the gantry as an organizer of social space and provider of services, makes it a socioeconomic asset to the entire community.

\section{Category 3: Appropriating Vehicular Infrastructure}

With precedent projects such as LeCorbusier's linear housing blocks running under a high speed roadway in his Plan Obus for Algiers, or Paul Rudolph's Lower Manhattan Expressway proposal in mind, two student teams developed designs that activate left over urban space at the edges of vehicular infrastructure. Median Strip by Azmiha Raza and Misbah Baig (figure 3) proposes situating a multi-functional linear building above the roadway median strip in Sharjah's industrial zones. The project combines affordable housing with a vocational training institution to provide workers improved access to educational opportunities which would thus enable the community in gaining better socio-economic mobility. By introducing a training campus in the industrial area, students sought to create more opportunities for a greater, more diverse workforce to come to UAE. The institution would also function as a place of communal gathering and recreation for the workers in the area. The ribbon is designed with a potential to expand and grow linearly along the vehicular infrastructure of the industrial zones, while contributing to the growth of Sharjah's economy by creating a stronger and more educationally advanced workforce.

Based on a critical urban analysis of Sharjah's industrial zones, specifically focused around mobility for vehicles and socio-economic mobility for workers, the Urban Loop Holes project by Jumanah Abbas and Sarah Adayleh (figure 4) is a proposal for greater symbiosis between architecture and infrastructure. By integrating housing and new public amenities within new layers of elevated roadways, the project not only improves vehicular mobility within Sharjah and Dubai, 

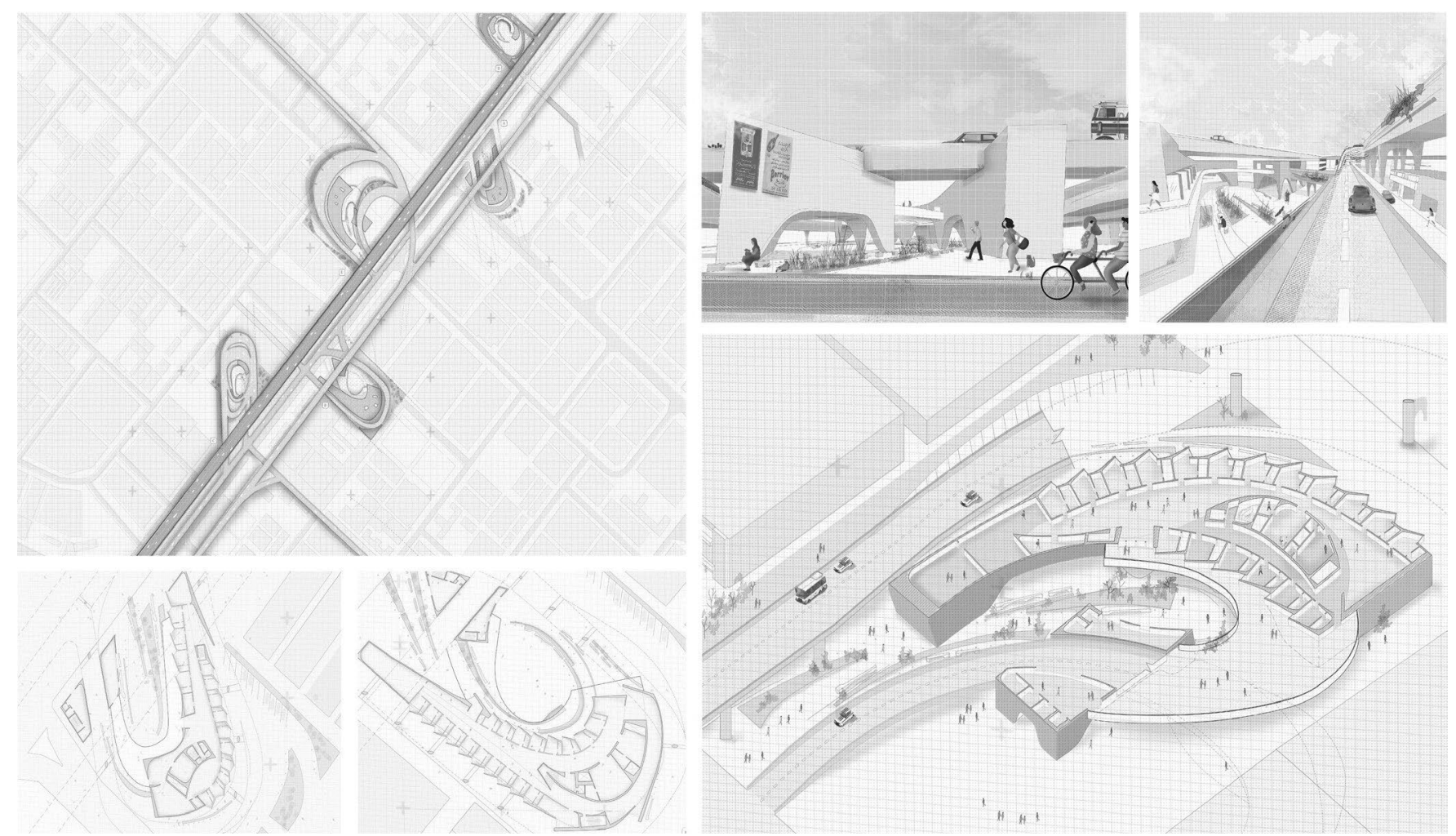

Figure 4: The Urban Loop Holes project by Jumanah Abbas and Sarah Adayleh introduces a new strand of vehicular infrastructure to better connect Sharjah with Dubai. It provides new spaces for affordable housing and public amenities in the curving loops of the highway offramps.

but uses a large-scale investments in vehicular infrastructure as a platform to offset the cost of social infrastructure. By elevating roadways and embedding looping, highway off-ramps into new buildings on vacant industrial lots, the project is able to reclaim the existing ground level as public space. Situated above, below or adjacent to the roadways, the architectural geometry and building typology of the housing is adapted to the curvature of the highway off-ramps. Considering the project at a global scale, Urban Loop Holes provides a new design methodology to integrate architecture with vehicular infrastructure that could be deployed in similar urban conditions across the world.

\section{Category 4: Anticipating Future Economies}

The students who developed this last category of projects made predictions about the social and economic development of Sharjah, Dubai and the Gulf region. Projects used governmental policies, newspaper articles and white papers concerning the growth of the Emirates as a research platform to shape their proposals. Subsequent projects examine how speculative planning for the present and future needs of a city might consider factors such as global tourism or industrial automation as drivers of urban development.

The project entitled Architectural Afterlife, by Amine El Kaddouri, was inspired by a case study of a modular, high-rise tower built out of self-structural, prefabricated components in China. In consideration of the context of Sharjah, a similarly modular project is proposed based on an analysis of the current condition of the industrial zones and assumptions about the future economy of the Emirate. Prefabricated housing units are built to satisfy present-day housing demand and reassembled at a future date in the form of a high-rise hotel for a developing tourism industry. Modular units come in a limited set of sizes and volumes and are used for both the low-rise, affordable worker housing units and the hotel tower. Over its lifespan, the units of housing complexes are imbued with subtle hints of the future and the hotel is marked with traces of the past. The project therefore transcends time and carries the past into the future. Each joint, mark of graffiti, crack, presence or absence of material typical of the old residential modular housing is reflected on the new assembly. The remnants of daily life and history of the city are carried over to the architecture of the future.

Shaimaa Mostafa Genidy's project, Housing Mass Tourism, is built off of one of Sharjah's strategic plans for economic development and global recognition, to attract more than ten million tourists to the Emirate per year by 2021 . The project proposes to build support system for the growing tourism industry by providing new parking platforms, strategically placed adjacent to existing sight-seeing routes for a fleet of tour buses. A new elevated datum, hovering above the existing industrial landscape, doubles as a space for affordable 


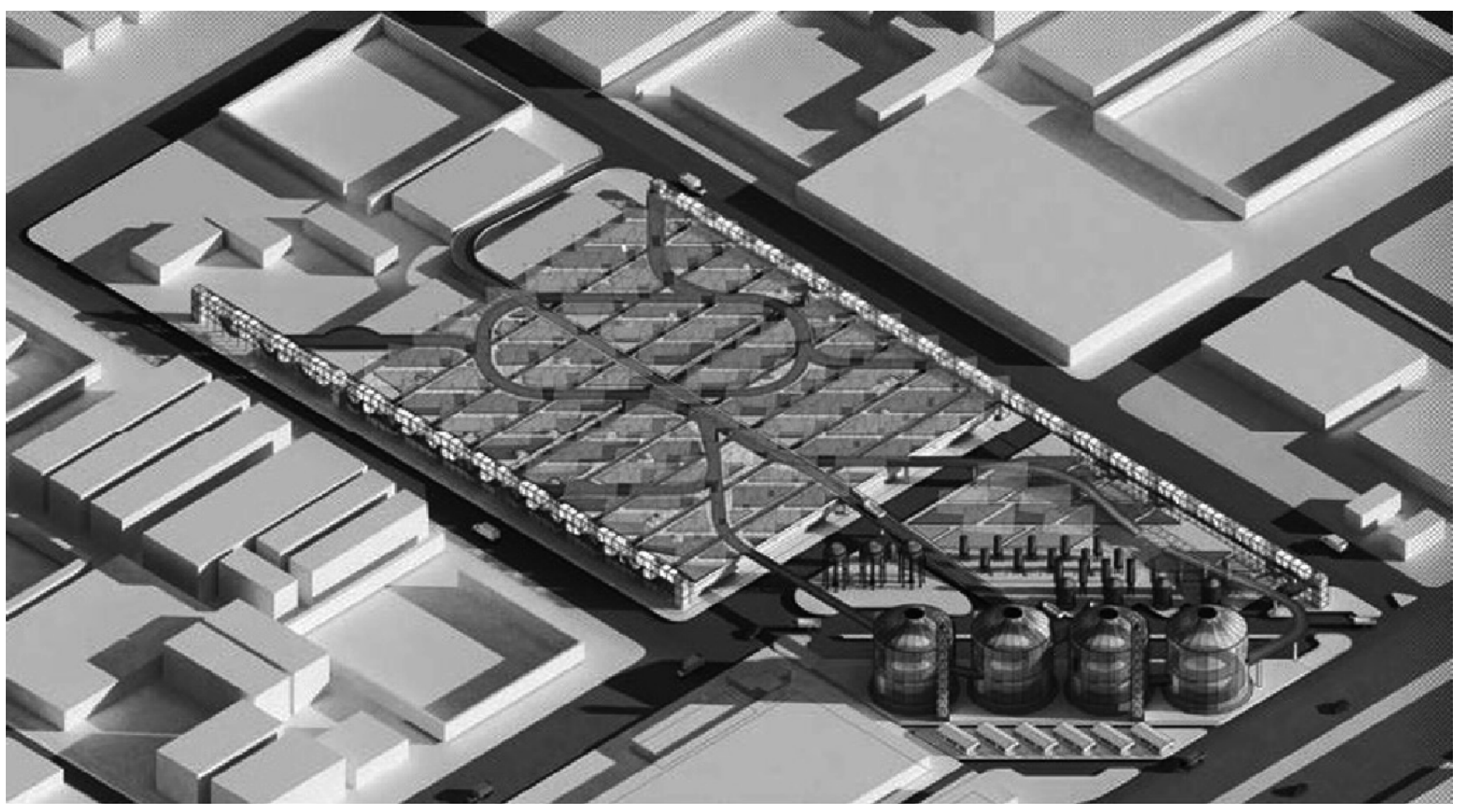

Figure 5: Agri-Industrial Housing, by Tasnim Tinawi and Uthra Varghese proposes rooftop farming, leisure zones and market spaces layered on top of existing worker housing, with new market housing at the periphery.

housing and public amenities together with a new home for the by-products of mass-tourism. Inspired by the typical cross section of a pre-cast concrete vehicular viaduct, the five hundred meter long, corrugated structure merges inhabitation and infrastructure, housing and parking, people and vehicles through varying the profile of the folds. The folding serves to create inhabitable spaces with seamlessly-blended floor, wall and roof planes. The project creates spaces that accommodate different types of functions ranging from small housing units, to communal dining rooms, landscaped parks to decking for bus parking and cricket matches.

Agri-Industrial Housing, by Tasnim Tinawi and Uthra Varghese (figure 5), explores the layering of agriculture and new residential infrastructure within an industrial site to yield a rich urban environment that mixes space for housing and leisure with urban farming, storage and distribution. New, affordable housing units are situated within elevated rigid space frames along the edges of an existing worker housing complex. The new housing is positioned at the periphery of a new layer of rooftop farms which provide unexpected views that provide residents with a zone of respite from the industrial nature of the surrounding districts. Farming fields and shading devices follow a diagonal pattern that responds to the solar orientation of the site. The housing and adjacent farmers' market adopts a utilitarian architectural language by re-purposing storage silos as spaces for commercial activity. A sinuous path interrupts the gridded layout and provides circulation that connects the project. Through architectural intervention, the project seeks to elevate the socio-economic mobility of the residents by creating an independent agricultural industry where the workers farm their own food and sell their produce in the marketplace. The proposal for providing urban farming on the rooftops of existing housing aims to create a building type that can be later incorporated to other housing areas on site.

SHJ_2112 is a proposal that seeks to create a new urbanism that anticipates the constant changes that characterize rapidly developing cities such as Dubai and Sharjah. Designers Omar Badran and Pooja Annamaneni propose an architecture that evolves with time, one that will shape the urban economy today and one hundred years from now. The project addresses issues such as the inadequate standard of living that is available to individuals living and working within the industrial areas by attempting to enhance both living conditions and the supporting infrastructure of the city. Transforming over one hundred years, affordable housing evolves to become a new hub for Sharjah tourism and culture. The project correlates urban development with a projected master plan, through a series of economic milestones to eventually become a megastructure. Thin, pencil towers are built with small footprints across the active industrial zones of Sharjah provide affordable housing for the working class residents of Sharjah during the development of the Emirate over the next decades. Towers eventually are transformed into 
massive columns for a trade and culture oriented convention center as manual labor and industry is automated over time. The materiality of the project is modified by developments in building technologies over the next century. The architectural language is shaped by the physical, social and economic constraints of the existing site, allowing for activities within the area to continue and evolve, keeping in mind potential for future expansion and cultural evolution.

\section{CONCLUSIONS}

This studio for the design of new prototypes for affordable living developed in the studio challenged students to transcend typical housing types in the Dubai region by responding more thoughtfully to environmental, social, economic, temporal, urban and architectural conditions in Sharjah and beyond. Instead of focusing merely on the minimum spatial and economic requirements for affordability the studio work contends that only by increasing the scope and vision of housing to the scale of urban and regional infrastructure can architecture begin to provide public amenity and social mobility to affect flows of people and capital. Only through envisioning architecture at larger scales and across longer spans of time can designers start to reverse the trend towards inequality in extreme contexts such as Dubai and Sharjah.

\section{ENDNOTES}

1. "Dubai's housing policy for low-income people," opinion piece on the Gulf News official website, March 13, 2017, https://gulfnews.com/opinion/

2. Richard Sennett, "The Open City," in Berlin: Towards an Urban Age, 2006 p. 1, accessed June 14, 2018, https://Isecities.net/media/objects/articles/ the-open-city/en-gb/

3. Sennett, "The Open City," 1.

4. "Sponsoring Residency Visa by Expatriates," Government.ae, the official portal of the UAE Government, accessed July 23, 2018, https://www. government.ae/en/information-and-services/visa-and-emirates-id/ sponsoring-residency-visa-by-expatriates.

5. Afkar Abdullah, "Leave Family Areas, Sharjah tells Bachelors," Khaleej Times, October 30, 2017, 6.

6. Andrea Kahn, "Defining Urban Sites," in Site Matters, ed., Carol Burns and Andrea Kahn (London: Routledge, 2004), 286. 